\title{
Quality of Life in Organ Transplant Recipients Participating in an Online Transplant Community
}

\author{
Paul Wicks $\cdot$ Katherine A. Sulham • \\ Ari Gnanasakthy
}

Published online: 6 November 2013

(c) The Author(s) 2013. This article is published with open access at Springerlink.com

\begin{abstract}
Background The PatientsLikeMe Organ Transplants online community allows patients to share detailed health information for research.

Objectives The objectives of our study were to describe and contrast data collected through an online community with the broader organ transplant population.

Methods Quantitative data were examined with respect to basic demographic characteristics and quantitative data including treatment, symptoms, side effects, and the PatientsLikeMe Quality of Life (PLMQOL) scale. Qualitative data including forum discussion posts and treatment evaluations were examined to support future development of standardized questions that could be added to the platform. Online data were compared with US national registry data from the United Network for Organ Sharing (UNOS). Results Within 30 days of account creation, 1,924 singleorgan transplant patients provided spontaneous, patientreported data in the form of 915 reported symptoms, 938 treatment episodes, and 1,215 PLMQOL assessments. Relative to patients in the UNOS registry, online participants were more likely to be female, younger, and white. Lung transplant patients had worse quality-of-life scores than other organs. Average organ transplant quality-of-life scores were most similar to those of HIV patients, faring better than patients
\end{abstract}

P. Wicks $(\square)$

PatientsLikeMe, Inc., 155 Second St, Cambridge, MA 02141,

USA

e-mail: pwicks@patientslikeme.com

K. A. Sulham

The Medicines Company, 400 Fifth Ave, Waltham, MA, USA

A. Gnanasakthy

Novartis Pharmaceuticals Corporation, East Hanover, NJ, USA with epilepsy, fibromyalgia, mood disorders, Parkinson's disease, multiple sclerosis, or ALS. Site users generated 2,169 posts to 346 unique topic threads in the transplants forum. Conclusions Organ transplant patients are willing to report detailed health data through online communities across key domains-symptoms, treatment effects, and generic quality of life-that constitute the essential core of patient-reported outcomes. Patient-reported outcomes captured online have the potential to accelerate learning about patient experiences but suffer methodological challenges that must be overcome to maximize their utility.

\section{Key Points for Decision Makers}

- The Internet is no longer just where patients go to read leaflets or chat in forums, they are using the same tools used in clinical research studies to learn more about managing their disease and contribute to research.

- The number of patients online today is relatively small but is growing rapidly.

- Immediate access to patients who have received an organ transplant provides a different type of data to public health statistics or claims data—with an emphasis on the lived experience of disease.

- Encouraging patients to support one another to overcome their disease challenges could ultimately improve health outcomes.

- Online research platforms have strengths and weaknesses that suggest they should be deployed primarily for patients with serious long-term conditions, particularly those affecting females under the age of 60 years, in order to maximize engagement.

- Online platforms suffer from rapid attrition, which suggests key data should be captured at first visit. 


\section{Introduction}

Organ transplantation is a condition requiring life-long maintenance, and may dramatically impact a patient's quality of life [1]. The selection of candidates for transplant is contentious, so powerful data-collection mechanisms exist to monitor important long-term outcomes such as organ failure or death and improve the allocation of scarce organs through the United Network for Organ Sharing (UNOS). However, even after a successful transplant, patients face medical and personal challenges such as monitoring their health, adhering to medication, and coping with emotions related to their transplant such as guilt, fear, and responsibility, data that are not gathered easilytechnology may provide one solution.

Patients with chronic health conditions have been using email and online message boards for more than 30 years to have conversations in which they learn about their conditions and share information about or advocate for better care [2]. In more recent times, social networks such as Facebook have reduced the barriers to communicating online and drawing attention to health issues. Facebook itself recently added the ability for users to report their organ donor status, for example, and permits advocacy groups to raise awareness about the need for more donors.

Today, transplant recipients can use online message boards, such as the TransplantBuddies Forum (http://www. transplantbuddies.org) and its companion social network site TransplantFriends (http://www.transplantfriends.com). Social networks have already shown some early promise in supplementing traditional research methods. Mohammad and colleagues recently reported on long-term outcomes in pediatric liver transplant recipients treated in Chicago (IL, USA) between 1988 and 1992, where they used Facebook to contact young adults who were otherwise lost to followup as their contact details changed [3]. However, sites that permit only social sharing (e.g., text stories, photographs, and hyperlinks) stand in contrast to newer online communities that integrate data sharing and analysis of quantitative information about the patients' treatment and health (e.g., diagnoses, treatments, dosages, symptoms, and outcomes), such as 23andMe (http://www.23andme.com) and PatientsLikeMe (http://www.patientslikeme.com).

Originally launched for patients with amyotrophic lateral sclerosis (ALS) in 2006, today any patient may join PatientsLikeMe to share information, learn from others, and exchange support [4]. Patients self-report benefits such as improved health literacy, better communication with healthcare professionals, and development of a peer support network [5, 6], and efforts are underway to validate these objectively. The platform has also been proven useful in developing patient-reported outcomes (PROs), informed by the patient's own language and experience of disease
[7-9]. In 2010, PatientsLikeMe collaborated with Novartis Pharmaceuticals to develop and launch an online community for organ transplant patients. In this paper we describe key characteristics of the community, assess their quality of life relative to other patients using PatientsLikeMe, and consider the benefits and limitations of such a tool.

\section{Methods}

\subsection{Participants}

Patients reporting a single-organ transplant, limited to heart, lung, liver, kidney or pancreas, and who created accounts between 1 March 2010 and 31 December 2010 were eligible for study inclusion. The beginning date in this observation period is the date the site was opened for patients with organ transplants and the end date was chosen to allow comparison with other complete-year statistics. Patients can report multiple-organ transplants but the large number of possible permutations lead to groups too small to analyze here. Users are under no requirement to enter any data and they may choose to report as much data as they like according to any schedule, but can be prompted with optional reminders. Each potential data point may be reported both prospectively and retrospectively (e.g., symptoms, treatment starts and stops, treatment evaluations, lab test results, quality of life). To create a common baseline reference for comparisons among groups of patients defined by organ type, we examined the 'most recent' report offered by a patient within 30 days of the date they created their PatientsLikeMe account. Thus, here we describe the status of organ transplant patients at approximately the time they created a PatientsLikeMe account during the period 1 March 2010 through 31 December 2010.

Select demographic characteristics of US-based PatientsLikeMe members reporting a transplant in 2010 were compared with data collected through UNOS, publicly available through the Organ Procurement and Transplantation Network (OPTN). To contextualize transplant patients' quality of life we also compared the baseline quality of life reported by transplant patients during March-December 2010 with new members of PatientsLikeMe communities that existed during the same time period [epilepsy, fibromyalgia and myalgic encephalomyelitis (ME), HIV, mood disorders, multiple sclerosis, Parkinson's disease, and ALS] [6]. To address the question of attrition in online studies we report usage statistics.

\subsection{Quantitative Analysis}

Basic demographics (birth date, age, gender, race/ethnicity, highest educational attainment, and insurance status) and 
transplant history information (organ type, date of transplant, transplant center, co-morbid conditions, and donor characteristics) were solicited from each PatientsLikeMe user. Users also may choose to share more detailed information about the following:

- Lab values (including blood pressure, serum creatinine, and glomerular filtration rate, calculated using the Modification of Diet in Renal Disease (MDRD-4) equation [10])

- Symptoms

- Treatments (pharmacological and nonpharmacological)

- Quality of life per the PLMQOL

Pre-defined symptom surveys consisting of five primary symptoms (insomnia, depressed mood, pain, fatigue, and anxious mood) were available to all users, supplemented by organ-specific symptoms that were included on the basis of a literature review and clinician input. In addition to the pre-defined symptom lists, patients have the ability to add and monitor symptoms of importance to them-these added symptoms are coded against the Medical Dictionary for Regulatory Activities (MedDRA ${ }^{\circledR}$ ) and (if necessary) corrected for spelling errors or merged to duplicate terms by a health data integrity team at PatientsLikeMe. Patients can also input treatment information including start date, purpose, dosage, and end date, and have the option to add treatment evaluations by answering brief questions about perceived treatment efficacy, adherence, burden, and cost, with an open text field for comments and advice to other patients.

Quality of life is measured using the PLMQOL version 2 , a 24-item generic quality-of-life questionnaire developed for use on the site in order to avoid the costs and usage restrictions of a licensed instrument. The PLMQOL shares a similar conceptual framework to the widely used the RAND Corporation SF-36 [11] and contains 24 items mapped to three domains: physical (11 items), mental (8 items), and social (5 items). Psychometric validation demonstrated acceptable Cronbach's alpha for each subscale $(\alpha>0.8)$ and strong correlation between the relevant subdomains of the PLMQOL and the RAND SF-36 [physical function $(r=0.847, p<0.001)$, emotional wellbeing/mental $(r=0.842, \quad p<0.001)$, social function $(r=0.808, p<0.001)$ ] [12]. For this report, we calculated domain summary scores such that physical scores range from 0 to 44, mental scores from 0 to 32, and social scores from 0 to 20 . Higher scores indicate better quality of life.

For eligible patients, data in the above fields were examined and analyzed using SPSS ${ }^{\circledR}$ Statistics $\left(\right.$ IBM $^{\circledR}$, Armonk, NY, USA). Group differences were examined using Pearson's Chi-square test when comparing distributions and $F$-ratios when comparing mean differences.

\subsection{Qualitative Analysis}

Patients are able to interact with other PatientsLikeMe users by posting to threaded message forums, by leaving comments or questions on other user's profiles, or through the system's private messaging system. Qualitative data are also collected through an open text field in treatment evaluations. We systematically reviewed free-text collected from the PatientsLikeMe Organ Transplants Forum and spontaneously reported treatment evaluations to identify additional issues important to patients using PatientsLikeMe. For the purposes of this research, forum posts and treatment evaluations completed between 1 March 2010 and 31 December 2010 were harvested and analyzed using IBM $^{\circledR}$ SPSS $^{\circledR}$ Text Analytics for Surveys version 4.0

IBM $^{\circledR}$ SPSS $^{\circledR}$ Text Analytics uses linguistic-based text analysis algorithms to identify concepts (words or word groups) and types (semantic groupings of concepts) which are used by the analyst to build categories of responses for analysis and interpretation. Initial extraction of concepts and types produces a list and a frequency report of how many of the posts contain each concept or type. We reviewed the initial extract list for concepts that mapped into categories of patient experience that we found represented in the organ transplant PRO literature. These concepts worked as tags within the text analysis program to allow sorting and processing of the forum posts for further review.

\section{Results}

\subsection{Participants and Demographics}

A total of 3,057 patient accounts were created in the PatientsLikeMe Organ Transplants community between 1 March 2010 and 31 December 2010. Excluding patients who did not report information about a specific transplant and those reporting on multiple transplants, data from 1,924 single-organ transplant patients were included in the analysis (Fig. 1). Patient-reported date of transplant ranged from 10 December 1969 to 12 December 2010, with the median number of days transplanted prior to joining PatientsLikeMe ranging from 668 for pancreas recipients to 1,467 for kidney recipients.

Due to the tight controls and allocation mechanisms around organ donation, UNOS provides near-perfect data on 28,662 organ transplants performed in the USA in 2010. Table 1 provides a comparison of demographics between the PatientsLikeMe organ transplant 2010 incident cohort (patients reporting a transplant date between 1 January and 31 December 2010, $n=187$ ) and the larger population of 


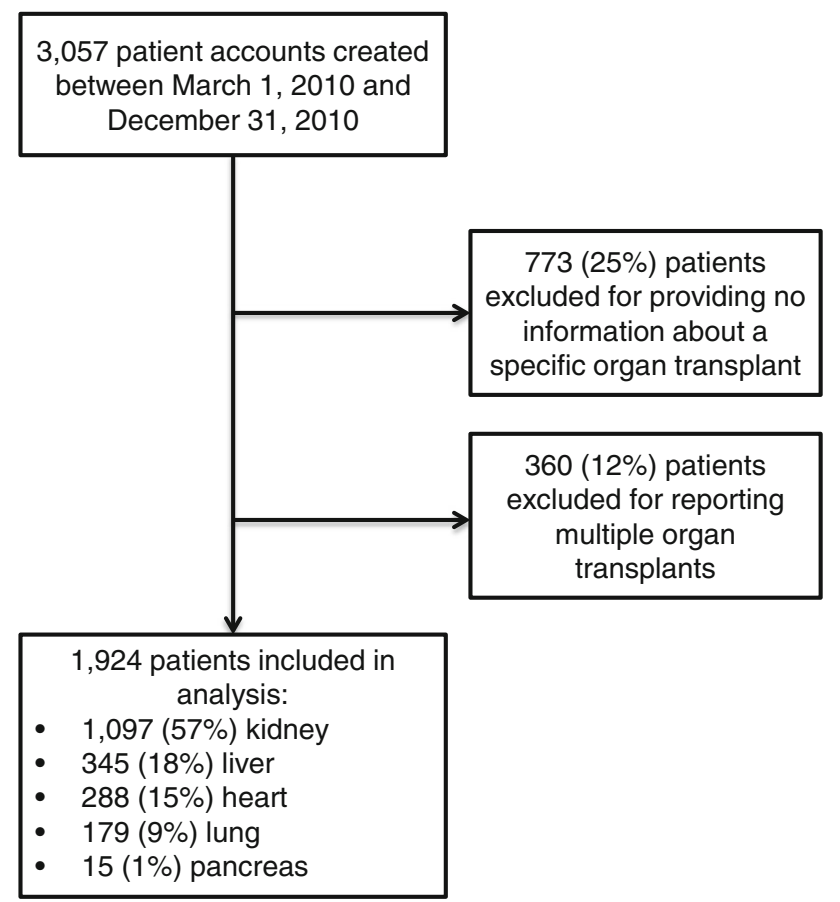

Fig. 1 Patient eligibility

Table 1 Demographics of United Network for Organ Sharing and PatientsLikeMe organ transplant recipients

\begin{tabular}{llll}
\hline Characteristic & $\begin{array}{l}\text { UNOS (US } \\
\text { transplants } \\
\text { performed in }\end{array}$ & $\begin{array}{l}\text { PLM (US } \\
\text { transplants }\end{array}$ & Significance \\
& $\begin{array}{l}\text { performed in } \\
\\
n=28,662)\end{array}$ & $2010, n=187)$ & \\
& & \\
\hline
\end{tabular}

\begin{tabular}{lccc}
\hline $\begin{array}{l}\text { Age }(\text { years })^{\mathrm{a}} \\
<18\end{array}$ & $1,827(6 \%)$ & $3(1 \%)$ & $\chi^{2}=81.839$, \\
$18-34$ & $3,225(11 \%)$ & $50(27 \%)$ & $p<0.001$ \\
$35-49$ & $6,898(24 \%)$ & $65(35 \%)$ & \\
$50-64$ & $12,420(43 \%)$ & $61(33 \%)$ & \\
$65+$ & $4,292(15 \%)$ & $4(2 \%)$ & \\
Gender & & & \\
Male & $17,878(62 \%)$ & $74(40 \%)$ & $\chi^{2}=38.191$, \\
Female & $10,784(38 \%)$ & $110(60 \%)$ & $p<0.001$ \\
Ethnicity & & & \\
White & $17,161(60 \%)$ & $137(73 \%)$ & $\chi^{2}=49.637$, \\
Black & $5,962(21 \%)$ & $11(6 \%)$ & $p<0.001$ \\
Hispanic & $3,796(13 \%)$ & $13(7 \%)$ & \\
Other & $1,743(6 \%)$ & $26(14 \%)$ & \\
\hline
\end{tabular}

Data are given as $n(\%)$

PLM PatientsLikeMe, UNOS United Network for Organ Sharing

${ }^{\text {a }}$ Four cases excluded from PLM dataset due to missing age data

b Three cases excluded from PLM due to missing gender data

c "Other" in UNOS includes: Asian, American Indian/Alaska native, Pacific Islander, multiracial, unknown. PLM race/ethnicity categories include: non-Hispanic white (categorized as White), non-Hispanic black (Black), Hispanic (Hispanic), other/unknown (Other) transplants performed in the USA in 2010 from UNOS $(n=28,662)$.

We found significant differences between UNOS and PatientsLikeMe users for age $\left(\chi^{2}=81.839, p<0.001\right)$, gender $\left(\chi^{2}=38.191, \quad p<0.001\right), \quad$ and ethnicity $\left(\chi^{2}=49.637, p<0.001\right)$. PatientsLikeMe users were less likely to be children ( $1 \%$ PatientsLikeMe vs. $6 \%$ UNOS) or seniors (2\% PatientsLikeMe vs. $15 \%$ UNOS), with most members being in early to middle adulthood. A higher proportion of PatientsLikeMe users were female $(59 \%)$ than in UNOS data (38\%), reflecting a female bias that has been detected in other conditions such as epilepsy or multiple sclerosis $[13,14]$. PatientsLikeMe users were more likely to be white (PatientsLikeMe $73 \%$ vs. UNOS $60 \%$ ) with lower proportions of black (PatientsLikeMe $6 \%$ vs. UNOS $21 \%$ ) and Hispanic (PatientsLikeMe $7 \%$ vs. UNOS $13 \%$ ) members but a higher proportion of users reporting "other" or "mixed race" (PatientsLikeMe $14 \%$ vs. UNOS $6 \%$ ).

In addition to those receiving a transplant in 2010, Table 2 provides additional detailed demographics of the total PatientsLikeMe single-organ transplant population, who might have received a transplant at any time in the past, by organ type. The average age of patients was significantly different across organs $\left(F_{4,1690}=5.23\right.$, $p<0.001)$ with kidney transplant patients the youngest [42.7 years, standard deviation (SD) 12.1] and lung transplant patients the oldest (46.6 years, SD 13.5). There were also between-group differences for gender $\left(\chi_{8}^{2}=47.16\right.$, $p<0.001)$, with a higher proportion of female lung recipients $(60 \%)$ relative to kidney $(55 \%)$, pancreas $(53 \%)$, liver $(49 \%)$, or heart (38\%). Comparisons with UNOS data were made difficult by a relatively high rate of missing data for gender in the PatientsLikeMe sample, around $10 \%$ of participants. By contrast, UNOS has only ever had a handful of transplants without gender being recorded. Comparison of adjusted gender ratios from PatientsLikeMe (disregarding the missing data) with 2010 data from UNOS continues to suggest a much higher proportion of females using the site. For example, $39 \%$ of UNOS kidney recipients were female compared with $63 \%$ (adjusted) of the PatientsLikeMe sample. There were more women than men in every organ group except heart transplants. Most patients resided in the USA, were nonHispanic, and white. Given that PatientsLikeMe is currently only in English and patient recruitment efforts are currently focused in the USA, this is not that surprising. The majority of patients reported at least some college education, and their health insurance is most likely to be private, employer-based, or Medicare.

Online communities and interventions are known to suffer from different forms of attrition, i.e., non-usage over time [15]. Within the sample reported here, patients logged 
Table 2 PatientsLikeMe demographic characteristics (users joining March-December 2010)

GED General Educational Development, $S D$ standard deviation, $V A$ Veterans Affairs

\begin{tabular}{|c|c|c|c|c|c|c|}
\hline & $\begin{array}{l}\text { Kidney } \\
(n=1,097)\end{array}$ & $\begin{array}{l}\text { Liver } \\
(n=345)\end{array}$ & $\begin{array}{l}\text { Heart } \\
(n=288)\end{array}$ & $\begin{array}{l}\text { Lung } \\
(n=179)\end{array}$ & $\begin{array}{l}\text { Pancreas } \\
(n=15)\end{array}$ & Statistical tests \\
\hline \multicolumn{7}{|l|}{ Age } \\
\hline Mean (SD) & $42.7(12.1)$ & $45.7(14.2)$ & $43.8(15.8)$ & $46.6(13.5)$ & $44.3(6.5)$ & $\begin{array}{c}F_{4,1,690}=5.23 \\
p<0.001\end{array}$ \\
\hline \multicolumn{7}{|l|}{ Gender (\%) } \\
\hline Female & 55 & 49 & 38 & 60 & 53 & \multirow{3}{*}{$\begin{array}{c}\chi_{8}^{2}=47.16 \\
p<0.001\end{array}$} \\
\hline Male & 33 & 41 & 50 & 32 & 13 & \\
\hline Not reported & 12 & 10 & 12 & 8 & 34 & \\
\hline \multicolumn{7}{|l|}{ Country (\%) } \\
\hline USA & 76 & 79 & 81 & 82 & 67 & \multirow{3}{*}{$\begin{aligned} \chi_{8}^{2} & =12.67 \\
p & =0.124\end{aligned}$} \\
\hline Other country & 13 & 11 & 8 & 11 & 7 & \\
\hline Not reported & 11 & 10 & 11 & 8 & 27 & \\
\hline \multicolumn{7}{|l|}{ Ethnicity (\%) } \\
\hline Hispanic & 5 & 4 & 4 & 1 & 7 & \multirow{3}{*}{$\begin{aligned} \chi_{8}^{2} & =19.89 \\
p & =0.011\end{aligned}$} \\
\hline Not Hispanic & 66 & 71 & 72 & 77 & 40 & \\
\hline Not reported & 29 & 25 & 24 & 22 & 53 & \\
\hline \multicolumn{7}{|l|}{ Race (\%) } \\
\hline White & 72 & 76 & 76 & 81 & 67 & \multirow{4}{*}{$\begin{array}{c}\chi_{12}^{2}=26.42 \\
p=0.009\end{array}$} \\
\hline Black & 7 & 3 & 4 & 4 & 0 & \\
\hline Other & 5 & 6 & 4 & 2 & 0 & \\
\hline Not reported & 16 & 15 & 16 & 13 & 33 & \\
\hline \multicolumn{7}{|l|}{ Education $(\%)$} \\
\hline 8th grade or less & 1 & 1 & 1 & 1 & 0 & \multirow{7}{*}{$\begin{aligned} \chi_{8}^{2} & =23.66 \\
p & =0.481\end{aligned}$} \\
\hline Some high school & 3 & 2 & 1 & 3 & 7 & \\
\hline $\begin{array}{l}\text { High school } \\
\text { graduate or GED }\end{array}$ & 13 & 13 & 16 & 17 & 0 & \\
\hline Some college & 35 & 39 & 37 & 37 & 47 & \\
\hline College graduate & 23 & 20 & 19 & 21 & 13 & \\
\hline $\begin{array}{l}\text { Postgraduate } \\
\text { degree }\end{array}$ & 9 & 9 & 9 & 7 & 0 & \\
\hline Not reported & 16 & 16 & 17 & 14 & 33 & \\
\hline \multicolumn{7}{|l|}{ Health insurance (\%) } \\
\hline Employer-based & 37 & 37 & 29 & 31 & 40 & \multirow{9}{*}{$\begin{array}{c}\chi_{36}^{2}=89.81 \\
p<0.001\end{array}$} \\
\hline Direct purchase & 2 & 7 & 3 & 4 & 0 & \\
\hline Medicare & 15 & 10 & 18 & 23 & 7 & \\
\hline Medicaid & 5 & 6 & 9 & 8 & 7 & \\
\hline $\mathrm{VA} /$ military & 2 & 2 & 5 & 1 & 0 & \\
\hline Other & 1 & 3 & 2 & 2 & 0 & \\
\hline $\begin{array}{l}\text { Ex-US national } \\
\text { insurance }\end{array}$ & 5 & 4 & 5 & 3 & 7 & \\
\hline No insurance & 4 & 2 & 2 & 0 & 0 & \\
\hline Not reported & 29 & 29 & 27 & 28 & 39 & \\
\hline
\end{tabular}

in a mean of ten times in their first 365 days on the site (SD 22 , median 5) and there were no significant differences in login frequency between organ types $\left(F_{4,1919}=0.701\right.$, $p=0.591)$. The distribution was highly skewed [7.325, standard error (SE) 0.056] with a high degree of kurtosis (68.847, SE 0.112); $80 \%$ of users logged in ten times or less in their first year on the site.

\subsection{Quantitative Patient-Reported Outcomes}

Within 30 days of account creation, 1,924 single-organ transplant patients completed 915 symptom reports (see Table 3). In addition to the prompted primary and supplemental symptoms, patients added an additional 121 symptoms of their own choosing. Symptoms added by 
Table 3 Primary symptoms by organ type: proportion of patients reporting moderate or severe symptoms

\begin{tabular}{|c|c|c|c|c|c|c|}
\hline & $\begin{array}{l}\text { Kidney } \\
(n=1,097)\end{array}$ & $\begin{array}{l}\text { Liver } \\
(n=345)\end{array}$ & $\begin{array}{l}\text { Heart } \\
(n=288)\end{array}$ & $\begin{array}{l}\text { Lung } \\
(n=179)\end{array}$ & $\begin{array}{l}\text { Pancreas } \\
(n=15)\end{array}$ & Statistical tests \\
\hline $\begin{array}{l}\text { Number of patients reporting within } 30 \text { days of } \\
\text { account creation, } n\end{array}$ & 511 & 188 & 128 & 80 & 8 & \\
\hline \multicolumn{7}{|l|}{ All organs $\mathrm{s}^{\mathrm{a}}$} \\
\hline Insomnia & $33 \%$ & $37 \%$ & $39 \%$ & $41 \%$ & $65 \%$ & $\begin{aligned} \chi_{4}^{2} & =8.70 \\
p & =0.069\end{aligned}$ \\
\hline Depressed mood & $15 \%$ & $21 \%$ & $17 \%$ & $16 \%$ & $63 \%$ & $\begin{aligned} \chi_{4}^{2} & =13.89 \\
p & =0.008\end{aligned}$ \\
\hline Pain & $16 \%$ & $21 \%$ & $16 \%$ & $28 \%$ & $25 \%$ & $\begin{aligned} \chi_{4}^{2} & =7.60 \\
p & =0.107\end{aligned}$ \\
\hline Fatigue & $36 \%$ & $46 \%$ & $30 \%$ & $37 \%$ & $65 \%$ & $\begin{aligned} \chi_{4}^{2} & =14.37 \\
p & =0.006\end{aligned}$ \\
\hline Anxious mood & $18 \%$ & $22 \%$ & $23 \%$ & $31 \%$ & $63 \%$ & $\begin{aligned} \chi_{4}^{2} & =17.39 \\
p & =0.002\end{aligned}$ \\
\hline \multicolumn{7}{|l|}{ Organ specific ${ }^{\mathrm{b}}$} \\
\hline Nausea or vomiting & $6 \%$ & & & & & \\
\hline Loss of appetite & $8 \%$ & & & & & \\
\hline Fluid retention & $14 \%$ & & & & & \\
\hline Itching $(n=267)^{\mathrm{c}}$ & $5 \%$ & & & & & \\
\hline Bruising & & $17 \%$ & & & & \\
\hline Jaundice & & $5 \%$ & & & & \\
\hline Drowsiness & & $21 \%$ & & & & \\
\hline Ascites & & $6 \%$ & & & & \\
\hline Shortness of breath & & & $9 \%$ & & & \\
\hline Problems concentrating & & & $17 \%$ & & & \\
\hline Persistent cough or wheeze & & & $8 \%$ & & & \\
\hline Fluid retention in lower extremities & & & $12 \%$ & & & \\
\hline Palpitations & & & $26 \%$ & & & \\
\hline Cough & & & & $21 \%$ & & \\
\hline Confusion & & & & $7 \%$ & & \\
\hline Restlessness & & & & $17 \%$ & & \\
\hline Rapid breathing & & & & $18 \%$ & & \\
\hline Abdominal pain & & & & & $0 \%$ & \\
\hline Indigestion & & & & & $25 \%$ & \\
\hline
\end{tabular}

${ }^{a}$ All patients report on these five primary symptoms when filling out a symptom report (symptoms are categorized as none, mild, moderate, or severe)

b Organ-specific symptoms included in symptom reports are based on literature review and clinician input

c "Itching" was added to the kidney transplant symptom report on 26 July 2010

five or more patients include anemia, high blood pressure, headache, dizziness, asthenia, tachycardia, leg cramps, cold sensations, hyperglycemia, corticosteroid-induced diabetes mellitus, migraine, non-Hodgkin lymphoma, candidiasis, night cramps in legs and feet, and hand tremors.

Symptom reports at the account creation baseline were provided by approximately $50 \%$ of patients. Examining the five primary symptoms common to all organ types, significant differences were observed for depressed mood, anxious mood, and fatigue, with pancreas and liver patients most likely to report their symptoms as moderate or severe. For example, there were significant differences in depressed mood by organ type $\left(\chi^{2}{ }_{4}=13.89, p=0.008\right)$, with pancreas patients reporting a much higher rate $(63 \%)$ than other organ types (15-21\%), although the relatively low sample in this group $(n=8)$ warrants caution. Moderate or severe insomnia was reported by at least one in three patients in each organ group, while moderate or severe pain was reported by at least one in six patients in each organ group; no significant differences between organ groups were observed. 
Table 4 Treatments and side effects

\begin{tabular}{|c|c|c|c|c|c|}
\hline & $\begin{array}{l}\text { Kidney } \\
(n=1,097)\end{array}$ & $\begin{array}{l}\text { Liver } \\
(n=345)\end{array}$ & $\begin{array}{l}\text { Heart } \\
(n=288)\end{array}$ & $\begin{array}{l}\text { Lung } \\
(n=179)\end{array}$ & $\begin{array}{l}\text { Pancreas } \\
(n=15)\end{array}$ \\
\hline $\begin{array}{l}\% \text { of patients reporting any treatments spanning } \\
\text { baseline account creation date }(n)\end{array}$ & $47 \%(513)$ & $56 \%(193)$ & $47 \%(135)$ & $51 \%(91)$ & $40 \%(6)$ \\
\hline $\begin{array}{l}\text { Median number of immunosuppressants patients } \\
\text { report currently taking (range) }\end{array}$ & $2(0-5)$ & $1(0-4)$ & $2(0-5)$ & $2(0-4)$ & $2(0-3)$ \\
\hline Median number of treatments patients report currently taking (range) & $3(1-24)$ & $2(1-14)$ & $2(1-24)$ & $4(1-33)$ & $2(1-5)$ \\
\hline $\begin{array}{l}\text { Number of patients reporting treatment side effects } \\
\text { during period from account creation to end of observation period }\end{array}$ & 12 & 6 & 4 & 3 & 0 \\
\hline
\end{tabular}

Table 5 Quality of life basic psychometric properties

Reliability is defined by Cronbach's alpha

$S D$ standard deviation

a Number of patients reporting quality of life after 11 May 2010, when the most recent version of the quality-of-life scale was released

\begin{tabular}{|c|c|c|c|c|c|c|}
\hline & $\begin{array}{l}\text { Kidney } \\
(n=1,097)\end{array}$ & $\begin{array}{l}\text { Liver } \\
(n=345)\end{array}$ & $\begin{array}{l}\text { Heart } \\
(n=288)\end{array}$ & $\begin{array}{l}\text { Lung } \\
(n=179)\end{array}$ & $\begin{array}{l}\text { Pancreas } \\
(n=15)\end{array}$ & Statistical tests \\
\hline$n^{\mathrm{a}}$ & 386 & 131 & 91 & 65 & 7 & \\
\hline \multicolumn{7}{|c|}{ Physical score $(0-4)$} \\
\hline Mean & 37 & 36 & 36 & 33 & 36 & \multirow{5}{*}{$\begin{array}{c}F_{4,675}=5.54 \\
p<0.001\end{array}$} \\
\hline SD & 7.1 & 7.1 & 8 & 10 & 9.7 & \\
\hline$\%$ floor & 0 & 0 & 0 & 0 & 0 & \\
\hline$\%$ ceiling & 21 & 17 & 17 & 9 & 29 & \\
\hline Reliability & 0.91 & 0.91 & 0.92 & 0.93 & 0.93 & \\
\hline \multicolumn{7}{|c|}{ Mental score $(0-32)$} \\
\hline Mean & 24 & 24 & 25 & 23 & 21 & \multirow{5}{*}{$\begin{array}{c}F_{4,675}=0.60 \\
p=0.664\end{array}$} \\
\hline SD & 6.7 & 6.4 & 7.3 & 7.5 & 9.9 & \\
\hline$\%$ floor & 0 & 0 & 1 & 0 & 0 & \\
\hline$\%$ ceiling & 10 & 12 & 19 & 14 & 14 & \\
\hline Reliability & 0.92 & 0.91 & 0.93 & 0.91 & 0.93 & \\
\hline \multicolumn{7}{|c|}{ Social score $(0-20)$} \\
\hline Mean & 15 & 15 & 15 & 13 & 12 & \multirow{5}{*}{$\begin{array}{c}F_{4,675}=2.32 \\
p=0.056\end{array}$} \\
\hline SD & 4.5 & 4.5 & 4.9 & 5.7 & 6.4 & \\
\hline$\%$ floor & 1 & 1 & 2 & 3 & 0 & \\
\hline$\%$ ceiling & 22 & 19 & 22 & 14 & 29 & \\
\hline Reliability & 0.84 & 0.85 & 0.86 & 0.91 & 0.91 & \\
\hline
\end{tabular}

Users also created a total of 938 unique instances of treatments taken within 30 days of account creation (Table 4). When patients report a treatment they are asked to indicate the start date (if known), purpose (e.g., "Prevent organ rejection"), dosage (where appropriate and prepopulated from a list of likely dosages from the Multum ${ }^{\mathrm{TM}}$ database), and stop date (where applicable). In addition, patients may choose to write an 'evaluation' of a treatment, resembling a consumer product review of their perceived efficacy, side effects, burden, advice for other patients, and reasons for discontinuation (if applicable). The average transplant patient reported taking two treatments for the purpose of preventing organ rejection; in addition, 25 patients voluntarily reported side effects associated with their treatments. Forty percent or more of patients did not enter treatment information within 30 days of account creation.

Finally, 1,215 quality-of-life (PLMQOL) assessments were completed. Data from 680 PLMQOL assessments completed post 11 May 2010 demonstrate strong reliability in each domain across organ types (Cronbach's alpha 0.84-0.91; Table 5). Comparison shows poorer physical scores for lung transplants than other organ groups. Comparison with new entrants in other PatientsLikeMe communities during the same time finds average quality-of-life scores most similar to those of HIV patients, and both groups generally faring better than patients with epilepsy, fibromyalgia and ME, mood disorders, Parkinson's disease, multiple sclerosis, or ALS (see Fig. 2; Table 6). The relatively better scores of organ transplant and HIV patients 
Fig. 2 Quality of life of patients reporting within 30 days of PatientsLikeMe account creation. All singleorgan transplants are compared with new entrants to existing PatientsLikeMe communities (higher scores represent better quality of life). Data from quality-of-life reports obtained between 11 May 2010 and 31 December 2011. ALS amyotrophic lateral sclerosis
All single organ transplants $(n=680)$

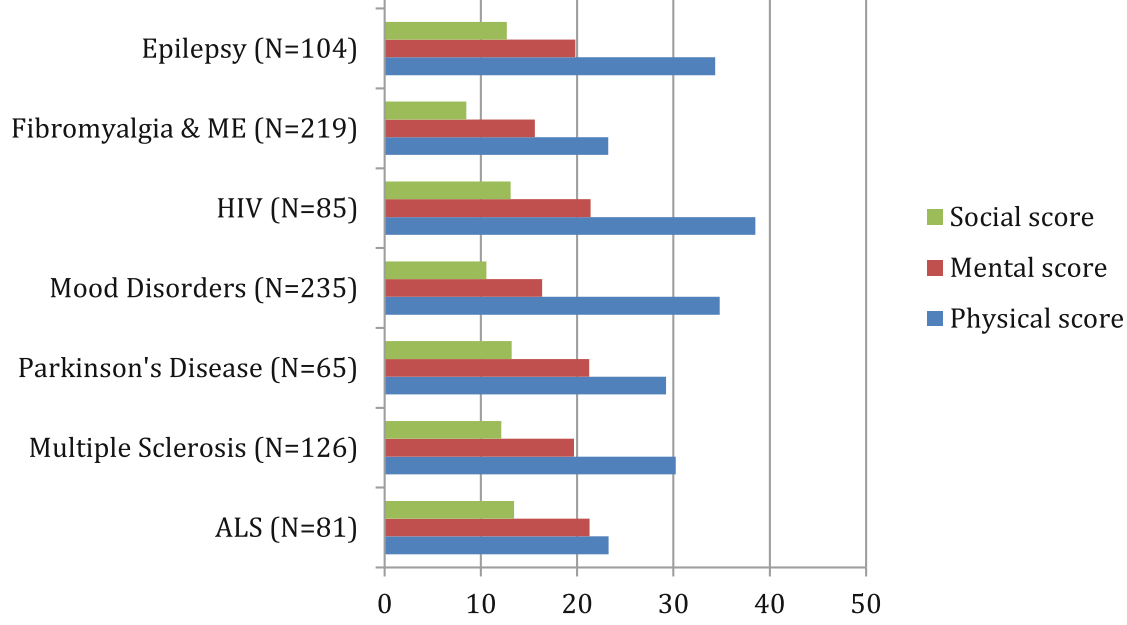

ME: Myalgic Encephalomyelitis, HIV: Human Immunodeficiency Virus, ALS: Amyotrophic Lateral Sclerosis Note: Data from Quality of Life reports obtained between May 11, 2010 and December 31, 2011. using PatientsLikeMe in comparison with the other disease groups may suggest such patients are benefitting from relatively effective long-term treatment regimens.

\subsection{Qualitative Review of Forum and Treatment Evaluations}

Between March and December 2010, there were 2,169 posts to 346 unique topic threads in the transplants forum. This content was contributed by 605 unique users (patients, caregivers, guests, and site administrators), 304 of whom made only one post. These one-off posts fell into the following categories: Introductory posts (e.g., "Hi I'm new here..."), questions (e.g., "Has anyone else had a gastric bypass?"), reply posts expressing similar experiences (e.g., "Susan, I've had that too..."), or posts offering information (e.g., "Try setting an alarm on your phone to remember meds"). These one-off posts were not qualitatively different from those made from members posting multiple times.

In total, 208 different users initiated the 346 forum threads, although a core of 47 users creating two or more threads accounted for $186(54 \%)$ of the threads. A total of 636 posts were not automatically classified; upon further review, 32 were forced into existing categories. A total of 1,565 posts were subject to further review. A single tag was applied to 692 of the posts, two tags were applied to 407 of the posts, and three or more tags were applied to the remaining 466 posts (Table 7). The most common tags included "organs," "symptoms," and "side effects", followed by "exercise and activity," "satisfaction," and "fear." Open-text analysis of 304 treatment evaluation entries reveals common tags of "symptoms," "side effects," and "compliance," closely mirroring results of the forum analysis (Table 8).
Table 6 Quality of life comparison of organ transplant with other PatientsLikeMe communities

\begin{tabular}{|c|c|c|c|}
\hline & Physical score & Mental score & Social score \\
\hline \multicolumn{4}{|c|}{$\operatorname{ALS}(n=81)$} \\
\hline Mean & 23 & 21 & 13 \\
\hline SD & 12.0 & 6.8 & 4.6 \\
\hline \multicolumn{4}{|c|}{ Multiple sclerosis $(n=126)$} \\
\hline Mean & 30 & 20 & 12 \\
\hline SD & 9.7 & 7.7 & 5.3 \\
\hline \multicolumn{4}{|c|}{ Parkinson's disease $(n=65)$} \\
\hline Mean & 29 & 21 & 13 \\
\hline SD & 10.8 & 6.3 & 5.1 \\
\hline \multicolumn{4}{|c|}{ Mood disorders $(n=235)$} \\
\hline Mean & 35 & 16 & 11 \\
\hline SD & 8.1 & 7.7 & 4.8 \\
\hline \multicolumn{4}{|c|}{ HIV $(n=85)$} \\
\hline Mean & 38 & 21 & 13 \\
\hline SD & 7.6 & 7.4 & 5.0 \\
\hline \multicolumn{4}{|c|}{ Fibromyalgia and ME $(n=219)$} \\
\hline Mean & 23 & 16 & 8 \\
\hline $\mathrm{SD}$ & 8.9 & 6.5 & 4.6 \\
\hline \multicolumn{4}{|c|}{ Epilepsy $(n=104)$} \\
\hline Mean & 34 & 20 & 13 \\
\hline $\mathrm{SD}$ & 8.5 & 8.1 & 5.1 \\
\hline \multicolumn{4}{|c|}{ All single-organ transplants $(n=680)$} \\
\hline Mean & 36 & 24 & 15 \\
\hline SD & 7.7 & 6.9 & 4.7 \\
\hline $\begin{array}{l}\text { Statistical } \\
\text { tests }\end{array}$ & $\begin{array}{c}F_{7,1,587}=82.14 \\
p<0.001\end{array}$ & $\begin{array}{c}F_{7,1,587}=47.92 \\
p<0.001\end{array}$ & $\begin{array}{c}F_{7,1,587}=50.38 \\
p<0.001\end{array}$ \\
\hline
\end{tabular}

Data from quality-of-life reports obtained between 11 May 2010 and 31 December 2011

$A L S$ amyotrophic lateral sclerosis, $M E$ myalgic encephalomyelitis, $S D$ standard deviation 
Table 7 Frequency of tag application to 1,565 categorized entries in the PatientsLikeMe forum

\begin{tabular}{|c|c|}
\hline Tag $^{\mathrm{a}}$ & $n$ \\
\hline Organs & 944 \\
\hline Symptoms & 403 \\
\hline Side effects & 298 \\
\hline Exercise and activity & 175 \\
\hline Satisfaction & 175 \\
\hline Fear & 170 \\
\hline Work life & 166 \\
\hline Family relationships & 166 \\
\hline Diet & 140 \\
\hline Weight & 119 \\
\hline Fatigue & 101 \\
\hline Mental health & 95 \\
\hline Pregnancy & 69 \\
\hline Gratitude & 66 \\
\hline Sleep & 44 \\
\hline Emotional & 35 \\
\hline Dysfunction & 29 \\
\hline Guilt & 28 \\
\hline Sex life & 20 \\
\hline Memory & 12 \\
\hline Medication Compliance & 9 \\
\hline Activities & 5 \\
\hline Physical well-being & 4 \\
\hline Concentration & 4 \\
\hline Social support & 3 \\
\hline Appearance & \\
\hline
\end{tabular}

${ }^{a}$ Multiple tags may be applied to any entry

\section{Discussion}

The organ transplant community on PatientsLikeMe provides tools to help patients share post-transplant experience among their peers. Within 9 months of launch, nearly 2,000 single-organ patients registered to use the system, and half of these provided data relating to their organ, quality of life, treatments, or symptoms. The use of structured and transplant-specific questions allowed comparison of the self-reported data to an established data source, UNOS/OPTN, while open-text narrative data allowed members to spontaneously report their concerns in their own patient voice.

Between March and December 2010, nearly $1 \%$ of all patients receiving an organ transplant in the USA joined PatientsLikeMe. That is not to say that this group was wholly representative, however, with users tending to be adults rather than children or seniors, with a higher proportion of females and whites than national data. The relatively low rate of use by children and seniors has been
Table 8 Frequency of tag application to 304 categorized advice entries in the PatientsLikeMe treatment evaluation system

\begin{tabular}{lr}
\hline Tag $^{\mathrm{a}}$ & $n$ \\
\hline Side effects & 143 \\
Symptoms & 68 \\
Medication Compliance & 50 \\
Organs & 34 \\
Sleep & 17 \\
Fatigue & 13 \\
Diet & 12 \\
Mental health & 9 \\
Appearance & 8 \\
Emotional & 6 \\
Activities & 4 \\
Fear & 4 \\
Satisfaction & 3 \\
Social support & 2 \\
Family relationships & 2 \\
Physical well-being & 1 \\
Gratitude & 1 \\
Sex life & 1 \\
\hline
\end{tabular}

${ }^{\text {a }}$ Multiple tags may be applied to any entry

observed previously when comparing PatientsLikeMe epilepsy users against insurance claims data [13]. It is likely that the site's focus on technically literate adult patients may be off-putting to parents of unwell children, who feel the site does not yet address their needs. Internal research is underway to better understand the needs of this group to help them find "ParentsLikeMe". Seniors might feel excluded by the requirement for Internet access and a relatively modern web browser, a digital divide that is likely to close over time. The different pattern of racial groups accessing the site might reflect different preferences for information seeking, marketing channels used to recruit patients, and, in the case of the relatively low proportion of Hispanic patients, the lack of availability of any language than English in the current version of the site. While some online communities such as TuDiabetes (http://www. tudiabetes.org) benefit from a Spanish-language version (EsTuDiabetes; http://www.estudiabetes.org), important issues such as adverse event reporting, safe and accurate communication between members, community moderation, and management of symptom terminology raise logistical barriers to multi-lingual sites.

While outcomes such as survival, rejection, hospitalization, and adverse events are collected routinely, less data have been collected with regard to quality-of-life outcomes. The reported experience of users of PatientsLikeMe, as in conventional quality-of-life assessment for transplant patients, reveals experience of symptoms that 
may relate to the functioning of the transplanted organ. For example, the following symptoms were reported as moderate or severe by $15 \%$ or more of patients at the time they joined the site: fluid retention in kidney patients, drowsiness in liver patients, palpitations in heart patients, various chest problems in lung patients, or indigestion in pancreas patients. We are also obtaining reports of symptoms associated with treatment, such as dizziness or hand tremors, but with low frequency as indicated by added symptoms and treatment adverse effect reports. The system includes features that permit spontaneously reported experiences to be quickly added to standard question items, ensuring the community continuously learns. There is even the potential for these data to be fed back into common ontologies to better inform the wider field [16].

Responses to the PLMQOL questions show that, on average, at baseline, users of the site were doing relatively well. Average scores in each of the three major domainsphysical, mental, and social-were toward the upper (good quality) end of the possible range, although as demonstrated by the ceiling estimates only 10-20\% (depending on organ type or domain) were responding that they were free of limitations due to their health. The data shown here indicate organ recipients as a group are doing better in each domain than patients with other significant life-altering conditions (Fig. 2).

PROs are gaining increasing prominence as useful tools in the measurement of medicine, for instance in detecting important outcomes such as fatigue in cancer [17], the detection of adverse events for marketed drugs [18], risk management programs for drugs with a high risk profile [19], and non-primary endpoints in the labels of newly approved drugs [20]. However, methodological limitations of PROs remain, such as unblinding, dealing with missing data, and cross-cultural validation of instruments. Online systems represent one method for experimentally addressing these limitations faster than traditional methods to boost the reliability and credibility of PROs with a view to exploring new uses they might one day have such as comparative effectiveness research.

In our experience, PRO research conducted online works best when a known, targeted group can be invited to participate in a study that directly addresses questions they are interested in, using brief cross-sectional surveys (less than 100 items is recommended), and with participants receiving immediate feedback on the data they contribute (such as where they lie in a distribution) in order to convey to them the value of participating in research. Response rates can be boosted with direct incentives (e.g., gift cards) or indirect incentives (e.g., donation to a non-profit) but are unlikely to rise to the response rate of questionnaires administered during a clinical visit. Longitudinal studies are difficult to maintain as it is easy for participants to ignore requests to participate, particularly when they come at arbitrary time points such as "three months later", rather than a meaningful trigger to the patient such as a change in their clinical status.

Much discussion exists around the advantages, disadvantages, and application of the Internet in the context of research [21, 22]. Key factors include condition, research question, and engagement. The most successful communities on our platform are for patients with conditions that are relatively rare, serious, with sufferers likely to be female and below the age of 60 years, and where the patient's actions and behaviors are likely to influence the outcome of their condition. Research questions that tap into the types of issues that patients spontaneously discuss with one another and complain about are more likely to yield greater response rates than questions of either purely academic or commercial interest. Online tools may be particularly useful in gaining rapid feedback where patient input is key, such as in the development of new PROs, where guidance from the FDA suggests this is a necessary component of scale validation [23]. PatientsLikeMe has recently announced the development of an "Open Research Exchange," which will allow PRO developers to prototype and test new PROs with users of the system willing to provide feedback and complete PROs in test phases to develop better instruments.

In moving from the conduct of research by human data collection to online studies there is an important consideration of engagement, an online form of rapport. In a traditional 'offline' clinical study, although there are (somewhat dry) informed consent documents to read through, a participant might also get the opportunity to meet an enthusiastic young researcher and have the ability to ask them other questions in order to learn more about their condition. In an online setting, however, this warmth, human interaction, and opportunity for serendipitous discovery is missing. In the online world these may be addressed in a different way, through design, but it is unclear the extent to which these will be important in maximizing response rates and value.

PatientsLikeMe aims to blend the power and 'stickiness' of social media and social networking with the rigors of structured observational data collection and analysis. In the context of organ transplantation, the Internet has historically been used as a mechanism to match donor organs with recipients. In addition, several websites exist that host forum capabilities and provide links to various transplantrelated resources. More broadly, websites and smartphone applications exist for patients with many health conditions, including organ transplant, to help individuals monitor their own health and medication information. PatientsLikeMe actively encourages capturing and sharing complex real-world information that is relevant to organ 
transplant, but also information that is relevant to understanding the patient context and experience.

This study may have implications for other conditions that involve surgical follow-up, such as prompting patients during the discharge period that they will receive an electronic follow-up at a relevant time point in the future and that, in the meantime, in addition to the support of their healthcare team, they will have access to the health experiences of other patients who have recovered from the same surgery. Because only a subset will be engaged longitudinally, it would be important to capture the most important PRO data in the first session visit in case the patient does not return. Future research will consider whether longitudinal attrition can be improved by integrating online data entry to a clinical visit.

This study had a number of limitations. Compared with the population-level data collected by UNOS, users of our system were found to be younger, more likely to be female, and more likely to be white. This may be reflective of the self-selecting nature of patients who come to the website and may affect the ability to generalize our findings. Inherent in any type of observational research is the potential for selection bias, information bias, and confounding, and such limitations may be amplified in research conducted via the Internet. Additionally, patients in our system can report retrospectively, which may be particularly prone to bias, hence our use of 2010 incident transplants in our comparative analysis. Some data, such as lab tests and dates of transplant and hospitalizations, may be accessible to patients in written or electronic records. However, retrospective PROs such as quality of life, symptom severity, or treatment evaluations may be less reliable. Unlike UNOS, we have no independent validation that patients are who they say or that they have truly received an organ transplant. However, the platform is policed by full-time moderators to look for signs of advertising or illegal services (such as attempting to 'sell' an organ), and technical measures are used to identify automated programs or attempts to register multiple accounts from the same source. A number of tools are also in place for users of the system to report suspicious activity to these moderators. There is currently little incentive for anyone to systematically enter falsified data, though this is a risk. Future research is underway to address means of independent validation that preserve some desirable elements of the platform, such as anonymity.

Finally, it is worth noting that organ transplantation has a unique resource in UNOS. In no other condition in the USA can the public access almost $100 \%$ complete, accurate, and up-to-date data on a medical condition. If such comprehensive data were available for every medical condition there would be no need for patients and researchers to develop innovative new methods to learn about one another.

\section{Conclusion}

Our initial description of this online community suggests significant opportunity in the space of PROs, symptoms, and side effects. However, there are also limitations of limited size, bias in the sample, attrition, and the nature of data that can be reported online. Integration of online communities into the transplant process could overcome some of these limitations and perhaps even yield other benefits.

Acknowledgments We are grateful to the members of our community for sharing their personal medical data openly.

Author contributions PW-Conception and design, analysis and interpretation of data, drafting the article, final approval. KASConception and design, acquisition of data, analysis and interpretation of data, revising the article, final approval. AG-Conception and design, revising the article, final approval.

Funding Disclosures This project was funded by Novartis Pharmaceuticals Corporation. The funders provided expert input and review in the design of the organ transplants community. Collection, analysis, and interpretation of the data was conducted by PatientsLikeMe with editorial input by the funder. The decision to submit for publication was made jointly.

PW is an employee of PatientsLikeMe, owns stock/options in the company, and is a named inventor on patents filed by PatientsLikeMe. The PatientsLikeMe R\&D team has received research support from Abbott, Acorda, Avanir, Biogen, Genzyme, Johnson \& Johnson, Merck, Novartis, Sanofi, and UCB.

KAS is a former employee of PatientsLikeMe. The PatientsLikeMe R\&D team has received research support from Abbott, Acorda, Avanir, Biogen, Genzyme, Johnson \& Johnson, Merck, Novartis, Sanofi, and UCB.

AG is an employee of Novartis Pharmaceuticals and holds stock/ options in the company.

Open Access This article is distributed under the terms of the Creative Commons Attribution Noncommercial License which permits any noncommercial use, distribution, and reproduction in any medium, provided the original author(s) and the source are credited.

\section{References}

1. Cleemput I, Dobbels F. Measuring patient-reported outcomes in solid organ transplant recipients: an overview of instruments developed to date. Pharmacoeconomics. 2007;25:269-86.

2. Slack WV. Cybermedicine: how computing empowers patients for better health care. Stud Health Technol Inform. 1998;52(Pt 1): 3-5.

3. Mohammad S, Hormaza L, Neighbors K, Boone P, Tierney M, Azzam RK, et al. Health status in young adults two decades after pediatric liver transplantation. Am J Transplant. 2012;12:1486-95.

4. Brownstein CA, Brownstein JS, Williams DS, Wicks P, Heywood JA. The power of social networking in medicine. Nat Biotechnol. 2009;27:888-90.

5. Wicks P, Keininger DL, Massagli MP, la Loge de C, Brownstein C, Isojärvi J, et al. Perceived benefits of sharing health data between people with epilepsy on an online platform. Epilepsy Behav. 2012;23:16-23. 
6. Wicks P, Massagli M, Frost J, Brownstein C, Okun S, Vaughan $\mathrm{T}$, et al. Sharing health data for better outcomes on PatientsLikeMe. J Med Internet Res. 2010;12:e19.

7. Wicks P, Massagli MP, Wolf C, Heywood J. Measuring function in advanced ALS: validation of ALSFRS-EX extension items. Eur J Neurol. 2009;16:353-9.

8. Wicks P, Massagli M, Kulkarni A, Dastani H. Use of an online community to develop patient-reported outcome instruments: the Multiple Sclerosis Treatment Adherence Questionnaire (MSTAQ). J Med Internet Res. 2011;13:e12.

9. Wicks P, Vaughan TE, Massagli MP. The multiple sclerosis rating scale, revised (MSRS-R): development, refinement, and psychometric validation using an online community [published erratum appears in Health Qual Life Outcomes. 2013;11:60]. Health Qual Life Outcomes. 2012 Jun 18;10:70.

10. Levey AS, Stevens LA, Schmid CH, Zhang YL, Castro AF, Feldman HI, et al. A new equation to estimate glomerular filtration rate. Ann Intern Med. 2009;5(150):604-12.

11. Hays RD, Sherbourne CD, Mazel RM. The RAND 36-item Health Survey 1.0. Health Econ. 1993;2:217-27.

12. Slawsky KA, Massagli MP, Wicks P. PRM30 a comparison of the Patientslikeme Quality of Life Questionnaire (PLMQOL) with the RAND SF-36. Value Health. 2011;14(7):A426.

13. la Loge de C, Keininger D, Isojärvi J. Characteristics of users of the epilepsy community of PatientsLikeMe.com and comparison with a representative claims database [abstract no. 1.305]. American Epilepsy Society; 2010. http://www.aesnet.org/files/ dmfile/Volume11Supplement1Abstracts2.pdf. Accessed 17 Oct 2013.

14. Bove R, Secor E, Healy BC, Musallam A, Vaughan T. Evaluation of an online platform for multiple sclerosis research: patient description, validation of severity scale, and exploration of BMI effects on disease course. PLoS One. 2013;8(3):e59707.

15. Eysenbach G. The law of attrition. J Med Internet Res. 2005;7:e11.

16. Doing-Harris KM, Zeng-Treitler Q. Computer-assisted update of a consumer health vocabulary through mining of social network data. J Med Internet. 2011;13:e37.

17. Barsevick AM, Cleeland CS, Manning DC, O'Mara AM, Reeve $\mathrm{BB}$, Scott JA, et al. ASCPRO recommendations for the assessment of fatigue as an outcome in clinical trials. J Pain Symptom Manag. 2010;39:1086-99.

18. Basch E, Abernethy AP, Mullins CD, Reeve BB, Smith ML, Coons SJ, et al. Recommendations for incorporating patientreported outcomes into clinical comparative effectiveness research in adult oncology. J Clin Oncol. 2012;1(30):4249-55.

19. Banerjee $A K$, Ingate $S$. Web-based patient-reported outcomes in drug safety and risk management: challenges and opportunities? Drug Saf. 2012;35:437-46.

20. Gnanasakthy A, Lewis S, Clark M, Mordin M, DeMuro C. Potential of patient-reported outcomes as nonprimary endpoints in clinical trials. Health Qual Life Outcomes. 2013;11:83.

21. Lefever S, Dal M, Matthíasdóttir Á. Online data collection in academic research: advantages and limitations. Br J Educ Technol. 2007;38:574-82.

22. Aanensen DM, Huntley DM, Feil EJ, al-Own F, Spratt BG. EpiCollect: linking smartphones to web applications for epidemiology, ecology and community data collection. PLoS One. 2009;4:e6968.

23. Basch E. Beyond the FDA PRO guidance: steps toward integrating meaningful patient-reported outcomes into regulatory trials and US drug labels. Value Health. 2012;15:401-3. 